\title{
Preoperative diffusion tensor imaging-fiber tracking for facial nerve identification in vestibular schwannoma: a systematic review on its evolution and current status with a pooled data analysis of surgical concordance rates
}

\author{
Amey R. Savardekar, MCh, Devi P. Patra, MD, MCh, Jai D. Thakur, MD, Vinayak Narayan, MD, MCh, \\ Nasser Mohammed, MD, MCh, Papireddy Bollam, MD, and Anil Nanda, MD, MPH \\ Department of Neurosurgery, Louisiana State University Health Sciences Center, Shreveport, Louisiana
}

OBJECTIVE Total tumor excision with the preservation of neurological function and quality of life is the goal of modernday vestibular schwannoma (VS) surgery. Postoperative facial nerve (FN) paralysis is a devastating complication of VS surgery. Determining the course of the FN in relation to a VS preoperatively is invaluable to the neurosurgeon and is likely to enhance surgical safety with respect to FN function. Diffusion tensor imaging-fiber tracking (DTI-FT) technology is slowly gaining traction as a viable tool for preoperative FN visualization in patients with VS.

METHODS A systematic review of the literature in the PubMed, Cochrane Library, and Web of Science databases was performed in accordance with Preferred Reporting Items for Systematic Reviews and Meta-Analyses (PRISMA) guidelines, and those studies that preoperatively localized the FN in relation to a VS using the DTI-FT technique and verified those preoperative FN tracking results by using microscopic observation and electrophysiological monitoring during microsurgery were included. A pooled analysis of studies was performed to calculate the surgical concordance rate (accuracy) of DTI-FT technology for FN localization.

RESULTS Fourteen studies included 234 VS patients (male/female ratio 1:1.4, age range 17-75 years) who had undergone preoperative DTI-FT for FN identification. The mean tumor size among the studies ranged from 29 to $41.3 \mathrm{~mm}$. Preoperative DTI-FT could not visualize the FN tract in 8 patients (3.4\%) and its findings could not be verified in 3 patients (1.2\%), were verified but discordant in 18 patients (7.6\%), and were verified and concordant in 205 patients (87.1\%). CONCLUSIONS Preoperative DTI-FT for FN identification is a useful adjunct in the surgical planning for large VSs (> $2.5 \mathrm{~cm}$ ). A pooled analysis showed that DTI-FT successfully identifies the complete FN course in $96.6 \%$ of VSs (226 of 234 cases) and that FN identification by DTI-FT is accurate in $90.6 \%$ of cases (205 of 226 cases). Larger studies with DTI-FT-integrated neuronavigation are required to look at the direct benefit offered by this specific technique in preserving postoperative FN function.

https://thejns.org/doi/abs/10.3171/2017.12.FOCUS17672

KEY WORDS vestibular schwannoma; acoustic neuroma; cerebellopontine angle tumor; diffusion tensor tractography; diffusion tensor imaging; facial nerve fiber tracking; preoperative facial nerve tractography; preoperative facial nerve imaging

A DVANCES in the surgical extirpation of vestibular schwannomas (VSs) have significantly improved the morbidity and mortality associated with the procedure. ${ }^{16,17}$ The goal of modern-day VS surgery is total excision of the tumor with the preservation of neurologi- cal function and quality of life. ${ }^{6}$ Postoperative facial nerve (FN) paralysis is one of the major complications of VS surgery and the most important determinant of quality of life. At present, VS surgery is associated with a rate of anatomical preservation of the FN that exceeds $95 \% .{ }^{22}$ Given

ABBREVIATIONS CE-FIESTA = contrast-enhanced FIESTA; CPA = cerebellopontine angle; $\mathrm{DTI}=$ diffusion tensor imaging; $\mathrm{DTT}=$ diffusion tensor tractography; FA = fractional anisotropy; FIESTA = fast imaging employing steady-state acquisition; FN = facial nerve; FT = fiber tracking; IAC = internal auditory canal; ROI = region of interest; VS = vestibular schwannoma.

SUBMITTED November 2, 2017. ACCEPTED December 11, 2017.

INCLUDE WHEN CITING DOI: 10.3171/2017.12.FOCUS17672. 
that surgery results in anatomical sacrifice of the $\mathrm{FN}$ in $5 \%$ of VS patients and that FN injury causes a sharp decrease in quality of life, attempts to preoperatively determine the course of the FN assume great significance. This is especially true for larger VSs (size $>3 \mathrm{~cm}$ ), which even experienced hands find challenging to achieve gross-total resection with successful preservation of FN function., ${ }^{1,6}$

The current strategy in VS surgery dictates early intraoperative identification of the FN based on both its relation to anatomical landmarks and electrical stimulation and electromyographic monitoring. 2,6 Any imaging study demonstrating the course of the FN preoperatively in VS patients should reasonably enhance surgical safety. If neuroimaging could preoperatively alert the neurosurgeon to any uncommon variation in FN displacement, unexpected injuries to the nerve could be avoided..$^{20}$ Identifying the FN course with traditional MRI sequences has been unreliable, especially for large VSs. Sartoretti-Schefer et al. evaluated the possibility of visualizing the FN on multiplanar reformatted 3D T2-weighted fast spin echo (FSE) images and found that it was only feasible in patients with VSs smaller than $25 \mathrm{~mm} \cdot{ }^{18}$ However, preoperative identification of the course of the FN in relation to large acoustic neuromas $(>2.5 \mathrm{~cm})$ with the help of diffusion tensor tractography (DTT) is slowly gaining traction and is being integrated into the lexicon of safe VS surgery. ${ }^{6,7,11,20}$ With technical refinements and improvements in imaging equipment, the boundary of what preoperative diffusion tensor imaging (DTI) is capable of achieving - and how it can aid in intraoperative FN localization-is constantly being pushed. ${ }^{15}$ We present a systematic review of the literature on the evolution and current status of DTT in preoperative imaging of the FN in patients with VS.

\section{Methods}

In this systematic review, we analyzed studies that had used DTI-fiber tracking (DTI-FT) for preoperative identification of the FN course in patients with VS. We pooled data from these studies and calculated the rate of intraoperative surgical concordance with preoperative DTI-FT findings. We developed a detailed protocol for the literature search, inclusion and exclusion criteria, and selection of studies. The Preferred Reporting Items for Systematic Reviews and Meta-Analyses (PRISMA) guidelines were followed. Two investigators (A.R.S. and D.P.P.) performed the literature search. To minimize selection bias, 2 authors (J.D.T. and V.N.) reviewed the articles independently. In cases of doubt, mutual consensus was reached after a discussion.

\section{Literature Search Strategy}

A literature search of titles and abstracts from January 1960 to June 2017 was conducted in the PubMed, Cochrane Library, and Web of Science databases. A combination of key-word searches was made to build a search strategy. Only English-language articles were searched. An independent Web search was made with the key words to include any unpublished literature. The key words used to build the search directory were "vestibular schwannoma," "acoustic neuroma," "cerebellopontine angle," "fa- cial nerve," "fiber tracking," "facial nerve tractography," "pre-operative facial nerve imaging," "diffusion tensor tractography," and "diffusion tensor imaging." Additional search terms were "surgery," "microsurgery," "diagnostic technique," and "monitoring." The process is detailed in Fig. 1.

\section{Inclusion Criteria}

Studies on preoperative FN localization in relation to a VS using the DTI-FT technique were included in the review. Only those studies that verified the preoperative FN tracking results by using microscopic observation and electrophysiological monitoring during microsurgery or neuronavigation were included in the statistical analysis for surgical concordance rates.

\section{Data Extraction}

Initially, relevant articles were retrieved in either title or abstract format, and full-text articles were subsequently collected for all original articles related to the study. All reference sections were manually reviewed and pertinent articles were identified. An extensive analysis was then performed to extract relevant clinical data such as patient age, sex, tumor size, MRI techniques, DTI-FT results, intraoperative verification findings, and postoperative House-Brackmann scores. The rate of surgical concordance with preoperative DTI-FT findings in each study was noted.

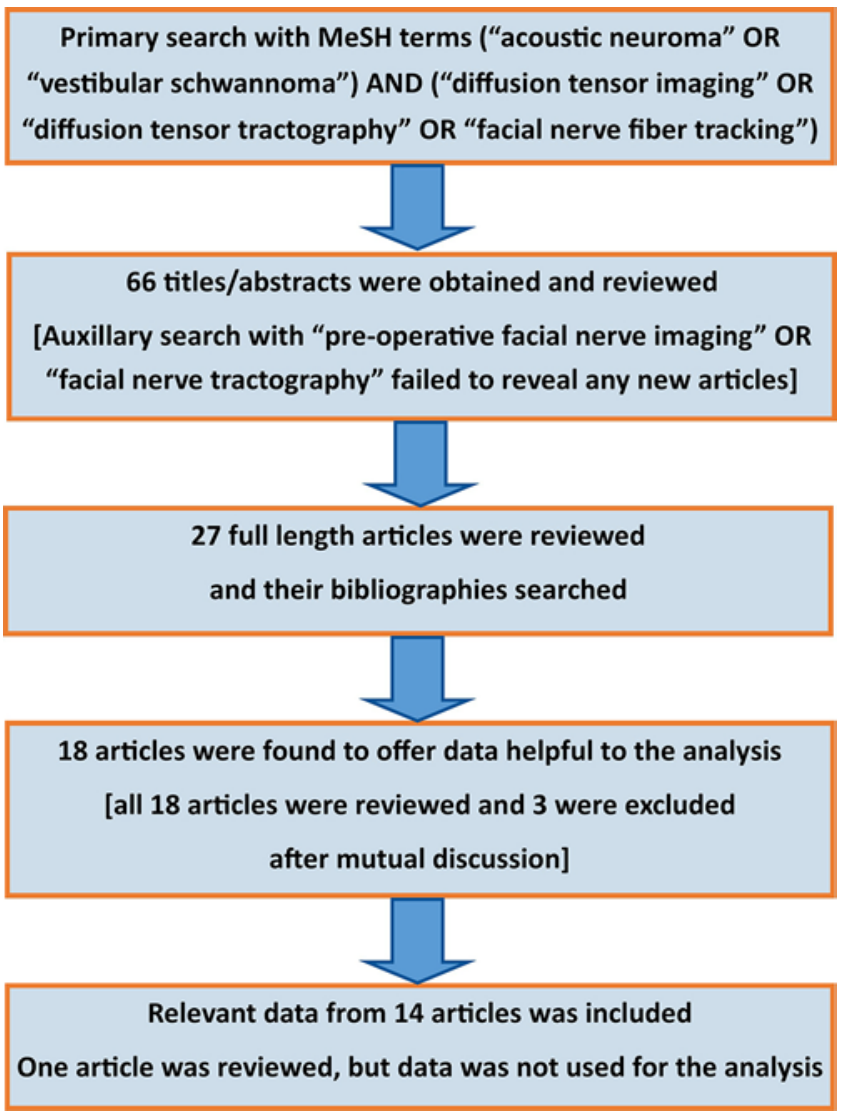

FIG. 1. Process of selection of final articles in the systematic review. 
TABLE 1. Characteristics of pooled patient population

\begin{tabular}{lcc}
\hline \multicolumn{1}{c}{ Parameter } & $\begin{array}{c}\text { No. of Patients } \\
\text { w/ Available Data }\end{array}$ & Value \\
\hline Total no. of patients & 234 & 234 \\
\hline Mean age in yrs (range) & 233 & $46.83(17-75)$ \\
\hline M/F & 232 & $1: 1.4$ \\
\hline Mean tumor diameter in $\mathrm{mm}$ (range) & 234 & $32.6(8.6-61)$ \\
\hline Solid/cystic tumors & 84 & $1: 3.6$ \\
\hline
\end{tabular}

\section{Results}

The primary search process yielded 66 articles, which were screened by title and abstract for relevance to the inquiry and usable data. Twenty-seven articles were selected in the secondary search, and the full text of these articles was carefully reviewed. Of these 27 articles, 14 reported on preoperative DTI-FT or tractography findings for FN tracking in VS and recorded the surgical concordance with the preoperative DTI-FT findings and thus were included in our analysis. The FN position was verified intraoperatively in a sector-wise manner (usually 6 sectors around the acoustic neuroma) in 13 articles. One study (Li et al. ${ }^{12}$ ) used DTI-integrated neuronavigation to verify the FN location. Four studies (among the 18 with data applicable to our analysis) were not ultimately considered in our analysis. One study (Ung et al. ${ }^{21}$ ) was a systematic analysis of FN DTI tractography studies that had been conducted in 2015 and included 3 studies. Another study (Chen et al. ${ }^{3}$ ) analyzed DTI for tracking the cranial nerves in VS but did not test the reliability of the results as compared with intraoperative findings. This study was included in our review, but the patient data were excluded from concordance analysis. The study by Nakai et al., ${ }^{14}$ published in 2013, involved preoperative detection of the FN via high-field MRI in VS patients but did not use DTI and hence was ex- cluded. A 2015 study by Yoshino et al. in 11 patients ${ }^{25}$ was excluded to avoid duplication of patients; this same group of authors reported on a larger series of 22 patients $^{24}$ later that same year, which included those previous 11 patients.

Fourteen published studies included 234 VS patients who had undergone preoperative DTI-FT for FN identification in relation to tumor. There were 96 males and 137 females (male/female ratio 1:1.4). Patients' ages ranged from 17 to 75 years. The VS size (maximum diameter) ranged from 9 to $61 \mathrm{~mm}$; however, the mean VS size ranged from 29 to $41.3 \mathrm{~mm}$. Tumor consistency was noted in only 6 studies, and the tumors among these studies were predominantly solid. The demographic profile and tumor characteristics in the 14 studies are shown in Table 1. The MRI machines, reconstructive software, and salient technical features are listed in Table 2.

Of the 234 VS patients who had undergone preoperative DTI for FN tracking, complete tracking of the nerve's course was obtained in 226 patients $(96.6 \%)$. Thus, the success rate for visualizing the FN via DTI-FT was $96.6 \%$ in our pooled analysis. Table 3 documents the DTI-FT FN identification rate, reasons for nonidentification, and the DTI-FT surgical concordance rates for all 14 studies. Surgical concordance with the preoperative DTI-FT findings for FN localization in VS was obtained in 205 patients (90.7\%). Thus, the accuracy rate of the FN visualization results of the DTI-FT technique was $90.7 \%$. Among the 234 VS patients in our pooled analysis, preoperative DTIFT could not visualize the FN tract in 8 patients $(3.4 \%)$ and its findings could not be verified in 3 patients (1.2\%), were verified but discordant with surgical findings in 18 patients $(7.6 \%)$, and were verified and concordant with surgical findings in 205 patients $(87.6 \%)$. The results of each study are detailed in Table 3.

\section{Discussion}

Surgery for VS in the present era is highly evolved,

TABLE 2. DTI protocol summary

\begin{tabular}{|c|c|c|c|c|c|}
\hline Authors \& Year & $\begin{array}{l}\text { Imaging Field } \\
\text { Strength }(T)\end{array}$ & $\begin{array}{c}\text { DTI Slice } \\
\text { Thickness (mm) }\end{array}$ & $\begin{array}{l}\text { DTI Diffuse } \\
\text { Directions }\end{array}$ & DTI Software & $\mathrm{FA}$ \\
\hline Taoka et al., 2006 & 1.5 & 3.0 & 6 & Masutani dTV & 0.1 \\
\hline Gerganov et al., 2011 & 3.0 & 1.6 & 12 & BrainLab iPlan & 0.1 \\
\hline Roundy et al., 2012 & 3.0 & 1.2 & 32 & Philips Intera \& TrackVis & 0.15 \\
\hline Zhang et al., 2013 & 3.0 & 3.0 & NA & NA & 0.1 \\
\hline Choi et al., 2014 & 1.5 & 2.5 & 32 & DTI-Studio software & $<0.15$ \\
\hline Wei et al., 2015 & 3.0 & 2.0 & 30 & BrainLab iPlan 3.03 & Variable \\
\hline Yoshino et al., $2015^{24}$ & 3.0 & 2.5 & 30 & FEl Avizo 6.3 \& Masutani dTV-II SR software & Variable \\
\hline Borkar et al., 2016 & 3.0 & 1.5 & 15 & Invivo DynaSuite Neuro 3.0 & $<0.12$ \\
\hline Hilly et al., 2016 & 3.0 & NA & 32 & Fibertrak module of Philips MRI extended workspace (version R2.6.1) & 0.1 \\
\hline Song et al., 2016 & 3.0 & 1.2 & 30 & 3D Slicer software & 0.1 \\
\hline Ma et al., 2016 & 3.0 & 2.0 & 32 & NA & 0.18 \\
\hline Zhang et al., 2017 & 3.0 & 2.0 & 64 & BrainLab iPlan 3.0 & Variable \\
\hline Zolal et al., $2017^{29}$ & 3.0 & 2.0 & 20 & FMRIB diffusion toolkit software & NA \\
\hline Li et al., 2017 & 3.0 & 1.0 & 32 & StealthViz software from Medtronic StealthStation system & Variable \\
\hline
\end{tabular}

$\mathrm{NA}=$ not available. 
TABLE 3. Results of DTI-FT and surgical concordance rates from series in the literature

\begin{tabular}{|c|c|c|c|c|c|c|c|c|}
\hline \multirow{2}{*}{$\begin{array}{l}\text { Authors } \\
\& \text { Year }\end{array}$} & \multirow{2}{*}{$\begin{array}{l}\text { Patient } \\
\text { Sample }\end{array}$} & \multicolumn{2}{|r|}{$\begin{array}{l}\text { DTI-FT Could Not } \\
\text { Show FN Tract }\end{array}$} & \multicolumn{2}{|c|}{$\begin{array}{l}\text { DTI-FT Findings Could } \\
\text { Not Be Verified }\end{array}$} & \multirow{2}{*}{$\begin{array}{l}\text { DTI-FT } \\
\text { Concordance } \\
\text { Verified (no.) }\end{array}$} & \multicolumn{2}{|r|}{ DTI Discordance Verified } \\
\hline & & No. & Reason Cited & No. & Reason Cited & & No. & Reason Cited \\
\hline $\begin{array}{l}\text { Taoka et al., } \\
2006\end{array}$ & 8 & 1 & $\begin{array}{l}\text { Smallest diameter (18 mm) } \\
\text { tumor (surrounding CSF } \\
\text { artifact) }\end{array}$ & 1 & $\begin{array}{l}\text { Intracapsular tumor } \\
\text { resection; hence, } \\
\text { FN not identified }\end{array}$ & 5 & 1 & Cystic nature of tumor \\
\hline $\begin{array}{l}\text { Gerganov et } \\
\text { al., } 2011\end{array}$ & 22 & 0 & & 0 & & 20 & 2 & $\begin{array}{l}\text { Large tumors w/ extremely thinned } \\
\quad \& \text { splayed out FNs }\end{array}$ \\
\hline $\begin{array}{l}\text { Roundy et } \\
\text { al., } 2012\end{array}$ & 5 & 0 & & 0 & & 5 & 0 & \\
\hline $\begin{array}{l}\text { Zhang et al., } \\
2013\end{array}$ & 8 & 1 & $\begin{array}{l}\text { Only distal portion of FN } \\
\text { could be mapped as } \\
\text { tumor was large ( } 48 \mathrm{~mm} \text { ); } \\
\text { FN attenuated \& indis- } \\
\text { tinguishable from capsule }\end{array}$ & 0 & & 7 & 0 & \\
\hline $\begin{array}{l}\text { Choi et al., } \\
2014\end{array}$ & 11 & 0 & & 0 & & 11 & 0 & \\
\hline $\begin{array}{l}\text { Wei et al., } \\
2015\end{array}$ & 23 & 0 & & 1 & $\begin{array}{l}\text { FN fiber too thin \& } \\
\text { could not be pre- } \\
\text { served or verified }\end{array}$ & 21 & 1 & $\begin{array}{l}\text { Technique failed to identify FN seg- } \\
\text { ment completely compressed } \\
\text { btwn tumor \& brainstem }\end{array}$ \\
\hline $\begin{array}{l}\text { Yoshino et } \\
\text { al., 2015 } 24 *\end{array}$ & 22 & 1 & Reason not mentioned & 0 & & 18 & 3 & Reason not mentioned \\
\hline $\begin{array}{l}\text { Borkar et al., } \\
\quad 2016\end{array}$ & 20 & 1 & Reason not mentioned & 1 & $\begin{array}{l}\text { FN could not be identi- } \\
\text { fied intraoperatively }\end{array}$ & 16 & 2 & Reason not mentioned \\
\hline $\begin{array}{l}\text { Hilly et al., } \\
2016\end{array}$ & 21 & 1 & $\begin{array}{l}\text { DTI could not track due to } \\
\text { splaying of fibers }\end{array}$ & 0 & & 18 & 2 & $\begin{array}{l}\text { Intraoperative findings suggested } \\
\text { that FNs significantly effaced } \\
\text { by tumor }\end{array}$ \\
\hline $\begin{array}{l}\text { Song et al., } \\
2016\end{array}$ & 15 & 1 & $\begin{array}{l}\text { Fasciculus was short \& } \\
\text { failed to be tracked }\end{array}$ & 0 & & 13 & 1 & $\begin{array}{l}\text { Protein structure of cyst fluid \& low } \\
\text { FA threshold value used }\end{array}$ \\
\hline $\begin{array}{l}\text { Ma et al., } \\
2016\end{array}$ & 9 & 1 & Reason not mentioned & 0 & & 8 & 0 & - \\
\hline $\begin{array}{l}\text { Zhang et al., } \\
2017\end{array}$ & 30 & 0 & & 0 & & 29 & 1 & $\begin{array}{l}\text { FN compressed by large tumor } \\
\text { \& was too thin; could not be } \\
\text { preserved }\end{array}$ \\
\hline $\begin{array}{l}\text { Zolal et al., } \\
2017^{29} \dagger\end{array}$ & 21 & 0 & & 0 & & 17 & 4 & $\begin{array}{l}\text { The } 4 \text { tumors significantly larger } \\
\text { than } 17 \text { tumors in which FN con- } \\
\text { cordance was achieved; also, } 2 \\
\text { were cystic }\end{array}$ \\
\hline $\begin{array}{l}\text { Li et al., } \\
2017 \ddagger\end{array}$ & 19 & 1 & $\begin{array}{l}\text { FN could not be tracked } \\
\text { around largest tumor } \\
(53.4 \mathrm{~mm}) \text { in the series }\end{array}$ & 0 & & 17 & 1 & Cystic tumor \\
\hline Total cases & 234 & 8 & & 3 & & 205 & 18 & \\
\hline
\end{tabular}

and complication rates are steadily decreasing because of constant improvement in neurosurgical expertise, surgeon experience, neuromonitoring techniques, and neuronavigation. ${ }^{6,16,17}$ Nevertheless, in large VSs, functional preservation of the $\mathrm{FN}$ - the most important determinant of postoperative quality of life - can be challenging even in the hands of experienced surgeons. ${ }^{6}$ As the tumor size increases, FN preservation becomes increasingly difficult ${ }^{1}$ because the FN in its cerebellopontine angle (CPA) segment is displaced by large VSs in an unpredictable manner and is often changed morphologically, making its identification particularly demanding for the surgeon. ${ }^{6}$ Although evoked potentials monitoring and careful microscopic inspections are performed to identify the compressed FN during surgery, an imaging-based technique that demonstrates the location of the nerve in relation to the VS preoperatively can be useful to the surgeon. Preoperative information regarding the exact course of the FN in relation to the tumor can change the choice of surgical approach for those surgeons who are just as comfortable 
with the retromastoid approach as with the translabyrinthine approach. ${ }^{7}$ It can also influence the sequential steps in terms of where to identify the nerve first and how to trace it safely until tumor resection is achieved.?

\section{Facial Nerve Imaging in Acoustic Neuroma}

When the tumor is moderately sized $(<2.5 \mathrm{~cm})$, the FN can be preoperatively identified using conventional MRI techniques such as MR cisternography, which produces high-resolution and strongly T2-weighted images. ${ }^{14,18}$ However, Sartoretti-Schefer et al. demonstrated that it is very difficult to visualize the course of the FN using conventional MRI techniques such as MR cisternography when the tumor is large $(>2.5 \mathrm{~cm})$ because of the focal nerve thinning and the obliteration of landmarks occurring within the internal auditory canal (IAC) and CPA..$^{18}$ The reasons for this are as follows: the signal intensities of the FN and VS are almost indistinguishable; focal FN thinning or effacement occurs because of mass effect by the VS; and, in large tumors, no cerebrospinal fluid intervenes between the two structures. ${ }^{20}$ Identification of the FN course in relation to the VS is a valuable adjunct to other techniques such as electrophysiological monitoring and neuronavigation in improving surgical outcomes, and the use of DTT for FN tracking has evolved as a reliable technique in this regard.

\section{Diffusion Tensor Imaging-Fiber Tracking for FN Localization}

Diffusion tensor tractography is a novel modality of MRI analysis that measures the diffusion direction of water molecules by combining multiple diffusion-weighted scans taken from multiple gradient directions. ${ }^{3}$ The diffusion of water molecules is thought to be anisotropic inside white matter tracts and therefore maximal along the direction of the fiber tracts. Diffusion tensor tractography allows the $3 \mathrm{D}$ reconstruction of the cranial nerves in healthy individuals. A 3D vector field (tensor) is assigned to each voxel. This information is then used to reconstruct and represent pictorially the white matter tracts within a specific region of interest (ROI). The reconstructed model of the fiber tracts is obtained in a highly reproducible manner. Diffusion tensor tractography has been widely used to trace several fibers such as the pyramidal tract, medial lemniscus, optic radiation, and so on. ${ }^{11}$ Hodaie et al. introduced DTT to cranial nerve tracking. ${ }^{9}$ Taoka et al. first reported the use of tracking the FN with DTT in patients with VS. ${ }^{20}$ Since then, several investigators have described their experience in applying this technique to reconstruct the FN tract in patients with VS. . $, 4,6,8,11,13,15,19,20,22,24,27-29$ Diffusion tensor tractography reconstruction of FN fibers is considered successful if a continuous tract of fibers is seen to extend from the internal auditory meatus to the brainstem along the tumor capsule. ${ }^{4}$

\section{Evolution and Current Status of DTI-FT for FN Tracking in VS}

Hodaie et al. studied the course of cranial nerves using DTI-FT in 4 healthy patients. ${ }^{9}$ The DTI-FT findings of the facial and vestibular complexes in this study primar- ily demonstrated the cisternal segment of these nerves; however, the authors could not clearly differentiate the facial, vestibular, and cochlear components. Taoka et al. first studied FN tracking with DTI-FT in 8 patients who underwent surgery for VS. ${ }^{20}$ A tract connecting the internal auditory meatus and brainstem was identified in 7 patients, and surgical concordance with the preoperatively determined FN course was confirmed in 5 patients.

Chen et al. successfully combined tractography and anatomical MRI using 3D Slicer software to demonstrate the precise location of the trigeminal nerve and FN in 3 patients with VSs; ${ }^{3}$ however, the DTI-FT findings were not verified intraoperatively in these 3 patients. The authors hypothesized that this technique could be useful in both intraoperative neuronavigation and radiosurgical planning to decrease injury to the cranial nerves. Gerganov et al. performed FN FT with DTI and anatomical images (constructive interference in steady state [CISS]) in a series of 22 consecutive patients with large VSs, and intraoperative concordance of the FN position with the DTI-based FT was noted in 20 (90.9\%) of the 22 patients. ${ }^{6}$ As compared with Taoka et al., ${ }^{20}$ in whose study slice spacing of $3 \mathrm{~mm}$ was used, Gerganov et al. used DTI with a $1.6-\mathrm{mm}$ thickness and no intersection gap. ${ }^{6}$ However, the DTI-based FT could not correlate the morphological types of the FN (compact or flat). ${ }^{6,20}$

Roundy et al. prospectively compared preoperative standard and high-density DTI in 5 patients with large (> $2.5 \mathrm{~cm}$ ) CPA tumors, which were mostly acoustic neuromas. ${ }^{15}$ Using standard DTI methods, these authors were unable to convincingly reconstruct the course of the FN in 4 of the 5 patients. However, using their high-density DTI method with more diffusion sensing directions and a smaller voxel size, they were able to accurately predict the entire course of the FN in each of the 5 patients, which was confirmed intraoperatively. This study was the first to use advanced high-density DTI techniques, and the authors attributed their $100 \%$ accuracy rate in FN identification to these advances in imaging technology.

The study by Wei et al. included 23 consecutive VS patients with Hannover classification T3b to T4b and in whom DTI and anatomical images were acquired. ${ }^{22}$ The aim of the study was to evaluate the effectiveness of this technique for identifying the FN, the cochlear nerve, and the trigeminal nerve. The authors proposed "superselective tracking" for differentiating nerve bundles. They used small ROIs to identify all single bundles that course from the brainstem to the IAC, which helped them in identifying distinct nerve fibers. They concluded that DTI-FT failed to identify the FN segment that was completely compressed between the tumor and brainstem and that this could be a limitation of DTI-FT.

Yoshino et al. studied 22 VS patients and assessed whether the combined use of DTI-FT and contrast-enhanced (CE) fast imaging employing steady-state acquisition (FIESTA) could improve the accuracy of predicting the courses of the FN and cochlear nerve. ${ }^{25,26}$ With DTIFT, depicted fibers running from the IAC to the brainstem were judged to represent the FN or vestibulocochlear nerve. With regard to imaging, the authors investigated multifused CE-FIESTA scans in which all 3D vessel 
models were shown simultaneously from various angles. The low-intensity areas running along the tumor from the brainstem to the IAC were judged to represent the FN or vestibulocochlear nerve. By using a combination of DTIFT and multifused CE-FIESTA, the authors were able to increase the number of VS patients for whom predicted results corresponded with the courses of both the FN and cochlear nerve. This was an advance in the comprehension of 3D operative anatomy. ${ }^{25}$

The studies by Wei et al. and Yoshino et al. demonstrated that superselective FT and setting the fractional anisotropy (FA) threshold at the upper limit may help to detect the FN and vestibulocochlear nerve; however, this capacity may be limited..$^{22,24,28}$ In a study of 30 patients, Zhang et al. combined superselective tracking and 3D FIESTA with cycled phase sequences (3D FIESTA-C) image integration, which enabled them to identify individual nerve bundles and to distinguish them from artifacts based on anatomical information provided by the 3D FIESTA-C images..$^{28}$ Additionally, to increase the tracking success rate, these authors set various FA threshold values for each patient. Thus, rather than setting one FA value, Zhang et al. gradually increased the FA threshold value by 0.01 from 0.06 to 2.0, thus allowing them to identify the appropriate FA threshold to obtain the primary tracking maps containing the most bundles that originated from the brainstem with the lowest density. The authors concluded that although DTI-FT was reliable for FN tracking in patients with large VSs, it may not allow one to distinguish the FN within the seventh and eighth cranial nerve complex. Gerganov et al. may have rightly argued that in large VSs, the vestibular nerves are partially or completely destroyed by the tumor and hence DTI-FT may not demonstrate these nerves effectively. ${ }^{6}$ As a result, DTI-FT findings (at present) in patients with large VSs may include the seventh and eighth cranial nerve complex. ${ }^{28}$ In the future, diffusion spectrum imaging can be used to distinguish the FN from the vestibulocochlear nerve and warrants further investigation..$^{23}$

Zolal et al. studied preoperative FN and cranial nerve positions in 21 patients with large VSs by using nontensorbased probabilistic tractography as compared to the standard tensor-based deterministic tractography..$^{30}$ Numerous studies have reported on the superiority of nontensorbased probabilistic tracking in the depiction of smaller fiber bundles, stating that the advantages of probabilistic FT seem to be attributable to better extraction of information about fiber direction in areas where multiple fiber populations occupy the same voxel, as is the situation in cranial nerves..$^{5,10,12}$ The probabilistic tracking showed a connection that correlated to the position of the FN in $81 \%$ of cases and to the position of the cochlear nerve in $33 \%$ of cases. Further studies comparing the accuracy of this technique with the standard tensor-based tractography are required.

Li et al. studied 19 VS patients to determine the effect of intraoperative FN DTI-integrated neuronavigation to verify the accuracy of preoperative DTI-FT. ${ }^{11}$ It was the first study to integrate DTI-FT of the FN into intraoperative neuronavigation. Prior to this, intraoperative verification relied on sector-wise location of the FN in relation to the VS based on microscopic observation and electro- physiological monitoring by the neurosurgeon. Intraoperative navigation confirmed DTI-FT accuracy in 17 (94.4\%) of the 18 patients in whom fiber tracts were visualized. This high rate of accuracy was in line with the accuracy of previous studies and could be considered as intraoperative validation of DTI-FT accuracy. The authors rightly stated that the most important factors influencing FN visualization results are the selection of the ROI and the set point of the FA threshold.

As noted above, multiple technical modifications in, along with the integration of imaging adjuncts into, the DTI-FT technology has resulted in its evolution to a state in which it may be considered a necessary tool for surgical planning in large VSs. Heros and Bhatia made a valid point in their editorial-that very experienced surgeons who have operated on hundreds of these tumors may not need this tool since, aided by experience with intraoperative stimulation, they have developed the ability to find, follow, and preserve the FN even in cases of large tumors. However, most VSs are not treated by surgeons who perform this operation several times a week, and for those surgeons without extensive experience, the ability to preoperatively predict with confidence the course of the FN in the capsule of the tumor would be valuable.?

\section{Conclusions}

Preoperative DTI-FT for FN identification is a useful adjunct in the surgical planning for large VSs $(>2.5 \mathrm{~cm})$. Our pooled analysis showed that DTI-FT successfully identifies the complete FN course in $96.6 \%$ of VSs (226 of 234 cases) and that FN identification by DTI-FT is accurate, with intraoperative validation in $90.6 \%$ of cases (205 of 226 cases). However, larger studies with DTI-FT-integrated neuronavigation are required to look at the direct benefit offered by this specific technique in preserving postoperative $\mathrm{FN}$ function.

\section{References}

1. Anderson DE, Leonetti J, Wind JJ, Cribari D, Fahey K: Resection of large vestibular schwannomas: facial nerve preservation in the context of surgical approach and patientassessed outcome. J Neurosurg 102:643-649, 2005

2. Borkar SA, Garg A, Mankotia DS, Joseph SL, Suri A, Kumar $\mathrm{R}$, et al: Prediction of facial nerve position in large vestibular schwannomas using diffusion tensor imaging tractography and its intraoperative correlation. Neurol India 64:965-970, 2016

3. Chen DQ, Quan J, Guha A, Tymianski M, Mikulis D, Hodaie M: Three-dimensional in vivo modeling of vestibular schwannomas and surrounding cranial nerves with diffusion imaging tractography. Neurosurgery 68:1077-1083, 2011

4. Choi KS, Kim MS, Kwon HG, Jang SH, Kim OL: Preoperative identification of facial nerve in vestibular schwannomas surgery using diffusion tensor tractography. J Korean Neurosurg Soc 56:11-15, 2014

5. Farquharson S, Tournier JD, Calamante F, Fabinyi G, Schneider-Kolsky M, Jackson GD, et al: White matter fiber tractography: why we need to move beyond DTI. J Neurosurg 118:1367-1377, 2013

6. Gerganov VM, Giordano M, Samii M, Samii A: Diffusion tensor imaging-based fiber tracking for prediction of the position of the facial nerve in relation to large vestibular schwannomas. J Neurosurg 115:1087-1093, 2011 
7. Heros RC, Bhatia R: Editorial. Vestibular schwannoma and the facial nerve. J Neurosurg 116:695-696, 2012

8. Hilly O, Chen JM, Birch J, Hwang E, Lin VY, Aviv RI, et al: Diffusion tensor imaging tractography of the facial nerve in patients with cerebellopontine angle tumors. Otol Neurotol 37:388-393, 2016

9. Hodaie M, Quan J, Chen DQ: In vivo visualization of cranial nerve pathways in humans using diffusion-based tractography. Neurosurgery 66:788-796, 2010

10. Khalsa S, Mayhew SD, Chechlacz M, Bagary M, Bagshaw AP: The structural and functional connectivity of the posterior cingulate cortex: comparison between deterministic and probabilistic tractography for the investigation of structurefunction relationships. Neuroimage 102:118-127, 2014

11. Li H, Wang L, Hao S, Li D, Wu Z, Zhang L, et al: Identification of the facial nerve in relation to vestibular schwannoma using preoperative diffusion tensor tractography and intraoperative tractography-integrated neuronavigation system. World Neurosurg 107:669-677, 2017

12. Li Z, Peck KK, Brennan NP, Jenabi M, Hsu M, Zhang Z, et al: Diffusion tensor tractography of the arcuate fasciculus in patients with brain tumors: Comparison between deterministic and probabilistic models. J Biomed Sci Eng 6:192-200, 2013

13. Ma J, Su S, Yue S, Zhao Y, Li Y, Chen X, et al: Preoperative visualization of cranial nerves in skull base tumor surgery using diffusion tensor imaging technology. Turk Neurosurg 26:805-812, 2016

14. Nakai T, Yamamoto H, Tanaka K, Koyama J, Fujita A, Taniguchi M, et al: Preoperative detection of the facial nerve by high-field magnetic resonance imaging in patients with vestibular schwannoma. Neuroradiology 55:615-620, 2013

15. Roundy N, Delashaw JB, Cetas JS: Preoperative identification of the facial nerve in patients with large cerebellopontine angle tumors using high-density diffusion tensor imaging. J Neurosurg 116:697-702, 2012

16. Samii M, Matthies C: Management of 1000 vestibular schwannomas (acoustic neuromas): surgical management and results with an emphasis on complications and how to avoid them. Neurosurgery 40:11-23, 1997

17. Samii M, Matthies C: Management of 1000 vestibular schwannomas (acoustic neuromas): the facial nerve-preservation and restitution of function. Neurosurgery 40:684695, 1997

18. Sartoretti-Schefer S, Kollias S, Valavanis A: Spatial relationship between vestibular schwannoma and facial nerve on three-dimensional T2-weighted fast spin-echo MR images. AJNR Am J Neuroradiol 21:810-816, 2000

19. Song F, Hou Y, Sun G, Chen X, Xu B, Huang JH, et al: In vivo visualization of the facial nerve in patients with acoustic neuroma using diffusion tensor imaging-based fiber tracking. J Neurosurg 125:787-794, 2016

20. Taoka T, Hirabayashi H, Nakagawa H, Sakamoto M, Myochin K, Hirohashi S, et al: Displacement of the facial nerve course by vestibular schwannoma: preoperative visualization using diffusion tensor tractography. J Magn Reson Imaging 24:1005-1010, 2006

21. Ung N, Mathur M, Chung LK, Cremer N, Pelargos P, Frew A, et al: A systematic analysis of the reliability of diffusion tensor imaging tractography for facial nerve imaging in patients with vestibular schwannoma. J Neurol Surg B Skull Base 77:314-318, 2016

22. Wei PH, Qi ZG, Chen G, Hu P, Li MC, Liang JT, et al: Identification of cranial nerves near large vestibular schwannomas using superselective diffusion tensor tractography: experience with 23 cases. Acta Neurochir (Wien) 157:1239-1249, 2015

23. Yoshino M, Abhinav K, Yeh FC, Panesar S, Fernandes D, Pathak S, et al: Visualization of cranial nerves using highdefinition fiber tractography. Neurosurgery 79:146-165, 2016

24. Yoshino M, Kin T, Ito A, Saito T, Nakagawa D, Ino K, et al: Combined use of diffusion tensor tractography and multifused contrast-enhanced FIESTA for predicting facial and cochlear nerve positions in relation to vestibular schwannoma. J Neurosurg 123:1480-1488, 2015

25. Yoshino M, Kin T, Ito A, Saito T, Nakagawa D, Ino K, et al: Feasibility of diffusion tensor tractography for preoperative prediction of the location of the facial and vestibulocochlear nerves in relation to vestibular schwannoma. Acta Neurochir (Wien) 157:939-946, 2015

26. Yoshino M, Kin T, Ito A, Saito T, Nakagawa D, Kamada K, et al: Diffusion tensor tractography of normal facial and vestibulocochlear nerves. Int J CARS 10:383-392, 2015

27. Zhang Y, Chen Y, Zou Y, Zhang W, Zhang R, Liu X, et al: Facial nerve preservation with preoperative identification and intraoperative monitoring in large vestibular schwannoma surgery. Acta Neurochir (Wien) 155:1857-1862, 2013

28. Zhang Y, Mao Z, Wei P, Jin Y, Ma L, Zhang J, et al: Preoperative prediction of location and shape of facial nerve in patients with large vestibular schwannomas using diffusion tensor imaging-based fiber tracking. World Neurosurg 99:70-78, 2017

29. Zolal A, Juratli TA, Podlesek D, Rieger B, Kitzler HH, Linn $\mathrm{J}$, et al: probabilistic tractography of the cranial nerves in vestibular schwannoma. World Neurosurg 107:47-53, 2017

30. Zolal A, Sobottka SB, Podlesek D, Linn J, Rieger B, Juratli TA, et al: Comparison of probabilistic and deterministic fiber tracking of cranial nerves. J Neurosurg 127:613-621, 2017

\section{Disclosures}

The authors report no conflict of interest concerning the materials or methods used in this study or the findings specified in this paper.

\section{Author Contributions}

Conception and design: Savardekar. Acquisition of data: Savardekar, Thakur. Analysis and interpretation of data: Savardekar, Patra, Thakur, Narayan, Mohammed. Drafting the article: Savardekar, Patra. Critically revising the article: Nanda, Savardekar. Reviewed submitted version of manuscript: all authors. Approved the final version of the manuscript on behalf of all authors: Nanda. Statistical analysis: Patra. Administrative/technical/material support: Bollam. Study supervision: Nanda, Bollam.

\section{Correspondence}

Anil Nanda: LSU Health Sciences Center, Shreveport, LA. ananda@1suhsc.edu. 